OPEN ACCESS

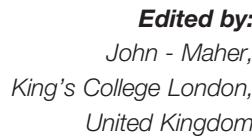

Reviewed by:

Wei Chen

Stanford University, United States Konnie Hebeda,

Radboud University Nijmegen Medical

Centre, Netherlands

${ }^{*}$ Correspondence:

Mariarita Sciumè

mariarita.sciume@policlinico.mi.it

Specialty section:

This article was submitted to

Cancer Immunity

and Immunotherapy,

a section of the journal

Frontiers in Oncology

Received: 30 June 2021 Accepted: 09 November 2021 Published: 30 November 2021

Citation:

Sciumè M, Ceparano G, Eller-Vainicher C, Fabris S, Lonati S, Croci GA, Baldini L and Grifoni FI (2021) Case Report: Evolution of

KIT D816V-Positive Systemic

Mastocytosis to Myeloid Neoplasm

With PDGFRA Rearrangement

Responsive to Imatinib.

Front. Oncol. 11:734025

doi: 10.3389/fonc. 2021.734025

\section{Case Report: Evolution of KIT D816V-Positive Systemic Mastocytosis to Myeloid Neoplasm With PDGFRA Rearrangement Responsive to Imatinib}

\author{
Mariarita Sciumè $^{1 *}$, Giusy Ceparano ${ }^{2}$, Cristina Eller-Vainicher ${ }^{3}$, Sonia Fabris ${ }^{1}$, \\ Silvia Lonati ${ }^{1}$, Giorgio Alberto Croci ${ }^{4}$, Luca Baldini ${ }^{1,2}$ and Federica Irene Grifoni ${ }^{1}$ \\ ${ }^{1}$ Hematology Unit, Fondazione Istituto di Ricovero e Cura a Carattere Scientifico (IRCCS) Ca' Granda Ospedale Maggiore \\ Policlinico, Milan, Italy, ${ }^{2}$ Postgraduate Medical School of Hematology, Università degli Studi di Milano, Milan, Italy, \\ ${ }^{3}$ Endocrinology and Diabetology Units, Department of Medical Sciences and Community, Fondazione Istituto di Ricovero e \\ Cura a Carattere Scientifico (IRCCS) Ca' Granda Ospedale Maggiore Policlinico, Milan, Italy, ${ }^{4}$ Division of Pathology, \\ Department of Pathophysiology and Transplantation, Fondazione Istituto di Ricovero e Cura a Carattere Scientifico (IRCCS) \\ Ca' Granda Ospedale Maggiore Policlinico, Milan, Italy
}

Systemic mastocytosis (SM) is a rare neoplasm resulting from extracutaneous infiltration of clonal mast cells (MC). The clinical features of SM are very heterogenous and treatment should be highly individualized. Up to $40 \%$ of all SM cases can be associated with another hematological neoplasm, most frequently myeloproliferative neoplasms. Here, we present a patient with indolent SM who subsequently developed a myeloid neoplasm with PDGFRA rearrangement with complete response to low-dose imatinib. The 63-year-old patient presented with eosinophilia and elevated serum tryptase level. Bone marrow analysis revealed aberrant MCs in aggregates co-expressing CD2/CD25 and KIT D816V mutation (0.01\%), and the FIP1L1-PDGFRA fusion gene was not identified. In the absence of ' $\mathrm{B}$ ' and ' $\mathrm{C}$ ' findings, we diagnosed an indolent form of SM. For 2 years after the diagnosis, the absolute eosinophil count progressively increased. Bone marrow evaluation showed myeloid hyperplasia and the FIP1L1-PDGFRA fusion gene was detected. Thus, the diagnosis of myeloid neoplasm with PDGFRA rearrangement was established. The patient was treated with imatinib $100 \mathrm{mg}$ daily and rapidly obtained a complete molecular remission. The clinical, biological, and therapeutic aspects of SM might be challenging, especially when another associated hematological disease is diagnosed. Little is known about the underlying molecular and immunological mechanisms that can promote one entity prevailing over the other one. Currently, the preferred concept of SM pathogenesis is a multimutated neoplasm in which KIT mutations represent a "phenotype modifier" toward SM. Our patient showed an evolution from KIT mutated indolent SM to a myeloid neoplasm with PDGFRA rearrangement; when the 
eosinophilic component expanded, a regression of the MC counterpart was observed. In conclusion, extensive clinical monitoring associated with molecular testing is essential to better define these rare diseases and consequently their prognosis and treatment.

Keywords: systemic mastocytosis, myeloid neoplasm with PDGFRA rearrangement, imatinib, KIT D816V mutation, clonal evolution

\section{INTRODUCTION}

Systemic mastocytosis (SM) is a heterogeneous group of neoplasms characterized by abnormal expansion of clonal mast cells (MCs) in the bone marrow (BM) and other extracutaneous organ-systems (1).

According to the World Health Organization (WHO) classification, the diagnosis of SM is established in presence of the major criterion and one minor criterion or at least three minor criteria. The major criterion is fulfilled by the detection of multifocal clusters of MCs (aggregates $\geq 15$ ) in one or more extracutaneous organs (usually $\mathrm{BM}$ ), while the minor criteria include aberrant MC expression of CD25 and/or CD2, abnormal morphology of MCs, KIT mutation $\mathrm{D} 816 \mathrm{~V}$, and a persistent serum tryptase level $\geq 20 \mathrm{ng} / \mathrm{ml}(1,2)$.

There are five subtypes of SM: indolent SM (ISM), smoldering SM (SSM), SM with an associated hematological (non-MC lineage) neoplasm (SM-AHN), aggressive SM (ASM), and mast cell leukemia (MCL) (1).

The diagnosis of ISM can be established if $<2 \mathrm{~B}$ findings and no $\mathrm{C}$ findings are detected; SSM is defined by $\geq 2$ ' $\mathrm{B}$ ' findings and no ' $\mathrm{C}$ ' findings. ASM is characterized by one or more $\mathrm{C}$ findings, while MCL is defined by MCs $\geq 20 \%$ on marrow smears $(1,2)$.

There are three types of ' $\mathrm{B}$ ' findings: $\mathrm{MC}$ infiltration $>30 \%$ on bone marrow biopsy and serum total tryptase $>200 \mathrm{ng} / \mathrm{mL}$; hepatomegaly with normal liver function, palpable splenomegaly without hypersplenism, and/or lymphadenopathy; signs of dysplasia or myeloproliferation in non-MC lineage. The six ' $\mathrm{C}$ ' findings are cytopenias; hepatomegaly with impairment of liver function, ascites, and/or portal hypertension; palpable splenomegaly with hypersplenism; malabsorption with weight loss due to gastrointestinal MC infiltrates; large osteolytic lesions $(1,2)$.

The goals of ISM treatment are symptom control, severe anaphylaxis prophylaxis, and osteoporosis treatment, while the advanced forms may require cytoreductive therapy. Historically, cytoreductive agents include interferon- $\alpha$ and cladribine. Allogeneic stem cell transplant could be considered in SMAHN when the associated hematologic neoplasm has an indication of transplantation and in relapsed/refractory ASM or acute MCL (2). With the advent of the tyrosine kinase inhibitors, many efforts have been made to find a proper inhibitor of SM KIT-driver mutation. Imatinib still plays a role in the treatment of rare SM cases that are KIT D816Vunmutated, while more recently midostaurin has been shown to induce major clinical responses in advanced SM regardless of KIT mutational status $(2,3)$.

Among the myeloid neoplasms, the WHO classification recognizes the family of the myeloid/lymphoid neoplasms with eosinophilia and rearrangement of PDGFRA, PDGFRB, and FGFR1, or with PCM1-JAK2. These are rare diseases characterized by a fusion gene or a mutation resulting in the expression of aberrant tyrosine kinases. Eosinophilia $\left(\geq 1.5 \times 10^{9} / \mathrm{L}\right)$ is one of the most common features of these neoplasms. In the subgroup associated with PDGFRA rearrangement, the most common genetic abnormality is the FIP1L1-PDGFRA gene fusion, caused by 4q12 deletion (Figure 1) (1, 4-6). Patients frequently complain of fatigue, pruritus, and symptoms related to eosinophilic infiltrates in different organs; splenomegaly and hepatomegaly are common findings (4-6).

The natural history of PDGFRA-rearranged neoplasms has been dramatically altered by imatinib and the dosage of $100 \mathrm{mg}$ daily could be sufficient to elicit a complete molecular response in most of the patients (7-9).

Herein, we report the clinical features and the management of a patient with KIT D816V-positive SM who subsequently developed a myeloid neoplasm with PDGFRA rearrangement with a complete molecular response to low-dose imatinib.

All procedures were in accordance with the ethical standards of the responsible committee on human experimentation (institutional and national) and with the Helsinki Declaration of 1975 , as revised in 2013.

\section{CASE REPORT}

A 63-year-old Caucasian male patient was referred to hematological investigation because of eosinophilia.

His medical history was unremarkable except for postinfectious glomerulonephritis in childhood and flushing episodes that started one year before the hematological evaluation. No pathological findings were reported at physical examination. Previous blood tests revealed a gradual increase of absolute eosinophils count over two years. Recent laboratory examinations showed white blood count (WBC) $8.2 \times 10^{9} / \mathrm{L}$ with absolute eosinophil count $2.2 \times 10^{9} / \mathrm{L}(27 \%)$, serum tryptase level $26 \mathrm{ng} / \mathrm{mL}$ (normal level $<5 \mathrm{ng} / \mathrm{mL}$ ), normal serum lactate dehydrogenase $(\mathrm{LDH})$, and normal liver and renal function.

A bone marrow biopsy detected $60 \%$ of cellularity, with myeloid hyperplasia, increased eosinophils and reticulin, and the presence of multifocal clusters of spindle-shaped MCs coexpressing CD25 and CD2 (>15 cells). Karyotype analysis was normal. Polymerase chain reaction (PCR) detected the D816V KIT mutation on bone marrow (variant allele frequency - VAF $0.01 \%$ ), while the FIP1L1-PDGFRA fusion transcript was absent.

Overall, these findings were consistent with a diagnosis of SM for the presence of the major criterion (BM MCs infiltrates) and 


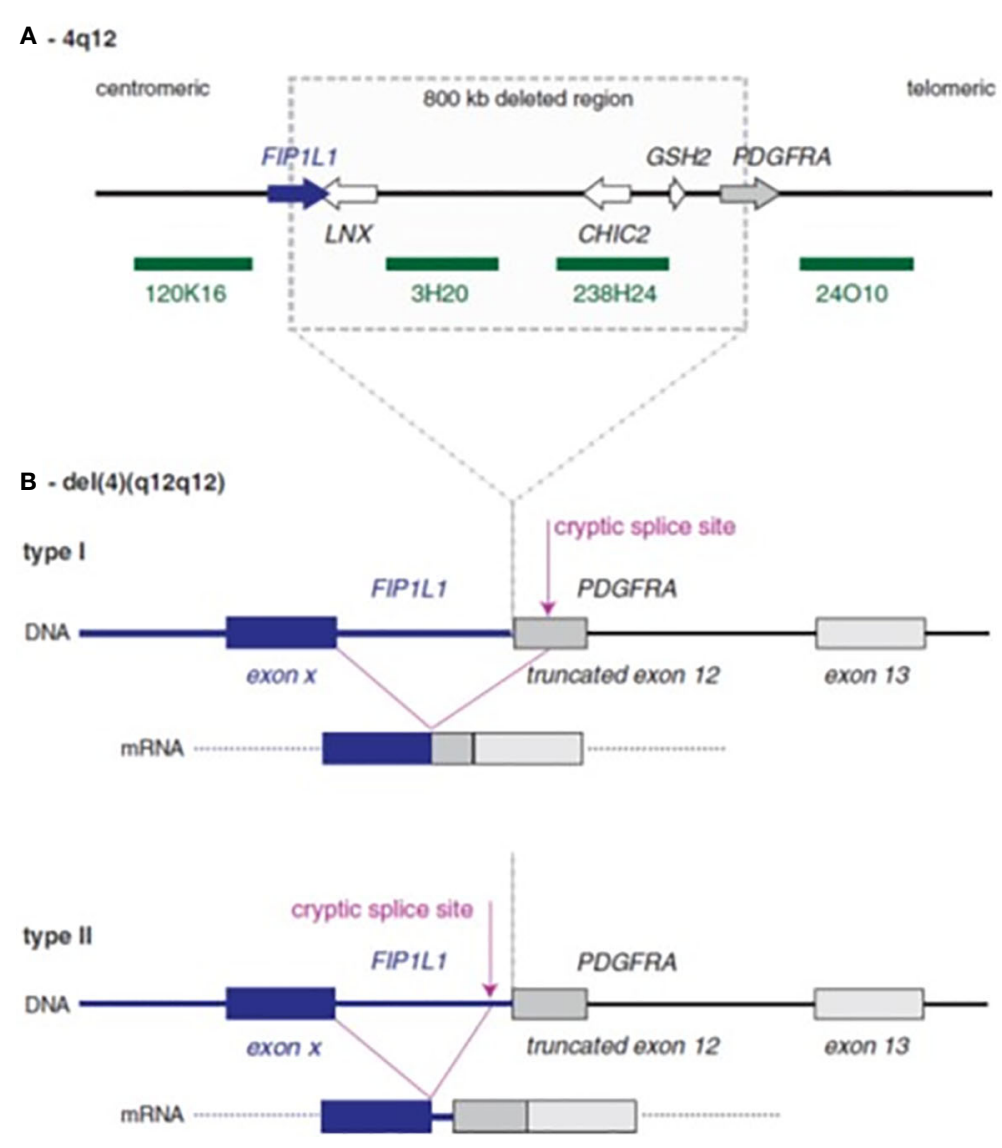

FIGURE 1 | Schematic representation of FIP1L1-PDGFRA rearrangement. (A) Normal 4q12 region with sites of deletion in cases with the FIP1L1-PDGFRA fusion. The four green bars denote probes that can be used to detect the deletion by fluorescence in situ hybridization. (B) The consequences of deletion: FIP1L1 usually breaks within an intron, while PDGFRA always breaks within exon 12. To obtain splicing between FIP1L1 and PDGFRA, cryptic splice sites need to be used, because the normal splice site in the exon 12 is removed by the deletion. This cryptic splice site could be located either within exon 12 of PDGFRA (type I fusion) or within the intron of FIP1L1 (type 2 fusion).

3 minor criteria (aberrant MCs, KIT D816V mutation, tryptase level $>20 \mathrm{ng} / \mathrm{mL}$ ). Abdominal ultrasound showed normal liver and spleen dimensions and an absence of lymphadenopathy. Dual-energy X-ray absorptiometry scan detected osteoporosis (lumbar T score -3.3, lumbar Z-score -2.6, femoral neck T-score -1.5 , femoral neck Z-score -0.5).

In the absence of ' $\mathrm{B}$ ' and ' $\mathrm{C}$ ' findings, we concluded with an indolent form of SM. The patient did not receive specific therapy other than osteoporosis treatment.

Two years later the patient complained of a worsening of flushing and a recurrent headache. Blood examinations revealed an increase of WBC $\left(13 \times 10^{9} / \mathrm{L}\right)$ with an absolute eosinophil count of $5.1 \times 10^{9} / \mathrm{L}$. Suspecting a myeloid neoplasm with eosinophilia, a new bone marrow evaluation was performed. The histological analysis confirmed myeloid hyperplasia with a marked increase of eosinophils and 3\% of MCs in rare aggregates. Aspirate smear revealed $36 \%$ of eosinophils and 6\% of MCs. No aberrant MCs were detected with flow cytometry. Cytogenetic analysis showed normal male karyotype and a digital PCR was negative for KIT D816V mutation. Real-time PCR performed on peripheral blood detected the FIP1L1-PDGFRA fusion transcript and serum tryptase was $20 \mathrm{ng} / \mathrm{ml}$. Thus, the final diagnosis was myeloid neoplasm with PDGFRA rearrangement.

Imatinib $100 \mathrm{mg}$ daily was started, and after 3 months all symptoms resolved and blood tests showed WBC 5.96×10 $/ \mathrm{L}$ with normal eosinophil count $\left(0.09 \times 10^{9} / \mathrm{L}\right)$.

$\mathrm{BM}$ analysis revealed the absence of eosinophils and the presence of rare MCs with normal morphology, corresponding to $4 \%$ of cellularity at the aspirate smear. Flow cytometry showed a normal MCs phenotype. FIP1L1-PDGFRA and KIT D816V mutations were negative on BM and peripheral blood.

A subsequent BM analysis, performed after 12 months of imatinib treatment, confirmed complete remission of the myeloid neoplasm, absence of aberrant $\mathrm{MCs}$, and negative FIP1L1-PDGFRA and KIT D816V mutations.

Table 1 and Figure 2 report the main hematological findings at diagnosis of SM, diagnosis of myeloid neoplasm with PDGFRA rearrangement, and after 3, 6, and 12 months of imatinib treatment. 
TABLE 1 | Relevant blood and bone marrow parameters in the main time-points from diagnosis of systemic mastocytosis.

\begin{tabular}{|c|c|c|c|c|c|}
\hline & SM Diagnosis & $\begin{array}{c}\text { Myeloid neoplasm } \\
\text { with PDGFRA } \\
\text { rearrangement } \\
\text { Diagnosis }\end{array}$ & $\begin{array}{l}\text { +3 mo of } \\
\text { Imatinib } \\
\text { Treatment }\end{array}$ & $\begin{array}{l}\text { +6 mo of } \\
\text { Imatinib } \\
\text { Treatment }\end{array}$ & $\begin{array}{l}\text { +12 mo of } \\
\text { Imatinib } \\
\text { Treatment }\end{array}$ \\
\hline WBC count, $\times 10^{9} / \mathrm{L}$ & 8.2 & 13.03 & 5.96 & 6.48 & 6.48 \\
\hline Eos. in blood, $\times 10^{9} / L(\%)$ & $2.2(27 \%)$ & $5.1(39.6 \%)$ & $0.09(1.5 \%)$ & $\begin{array}{c}0.15 \\
(2.3 \%)\end{array}$ & $0.18(2.8 \%)$ \\
\hline Serum tryptase, $\mathrm{ng} / \mathrm{mL}$ & 26 & 20 & & & 5 \\
\hline MCs in bone marrow, histological analysis, \% & $\begin{array}{c}\text { CD25+ CD2+, in aggregates, } \\
\text { not quantified }\end{array}$ & $3 \%$ & $2 \%$ & $1 \%$ & $1 \%$ \\
\hline MCs in bone marrow, aspirate smear, \% & Not performed & $6 \%$ & $4 \%$ & $0 \%$ & $0 \%$ \\
\hline MCs in bone marrow, flow cytometry, \% & Not performed & $0.3 \%$ & $2 \%$ & $1 \%$ & $0.2 \%$ \\
\hline $\begin{array}{l}\text { Genetic markers (mononuclear cells of peripheral } \\
\text { blood or bone marrow) }\end{array}$ & $\begin{array}{c}\text { Bone marrow: } \\
\text { PDGFRA - } \\
\text { KIT D816V + (0.01\%) }\end{array}$ & $\begin{array}{c}\text { Peripheral blood: } \\
\text { PDGFRA + } \\
\text { Bone marrow: KIT } \\
\text { D816V - }\end{array}$ & $\begin{array}{c}\text { Bone marrow and } \\
\text { peripheral blood: } \\
\text { PDGFRA - } \\
\text { KIT D816V - }\end{array}$ & $\begin{array}{c}\text { Bone } \\
\text { marrow: } \\
\text { PDGFRA } \\
- \\
\text { KIT } \\
\text { D816V - }\end{array}$ & $\begin{array}{l}\text { Bone marrow: } \\
\text { PDGFRA - } \\
\text { KIT D816V - }\end{array}$ \\
\hline
\end{tabular}

\section{DISCUSSION}

The clinical features of SM are very heterogenous, ranging from indolent forms to more aggressive diseases which require cytoreductive therapy $(1,2)$.

MCs derive from $\mathrm{CD} 34+/ \mathrm{CD} 117+$ pluripotent hematopoietic progenitor cells in BM. They are normal residents in mucosal tissues and skin with a key role in IgE-associated disorders and acquired or innate immunity (10). More than $90 \%$ of SM patients carried a somatic mutation in different regions of KIT which led to structural alteration of the protein with a constitutive activation of the receptor. The KIT D816V mutation is the most common (11). KIT median VAF strongly correlates with disease activity as represented by serum tryptase level, disease subtype (indolent versus advanced), and survival (12).

Up to $40 \%$ of all SM cases are associated with another hematological disease, which rarely can be a myeloid/lymphoid neoplasm with eosinophilia $(2,4)$.

Myeloid/lymphoid neoplasms with eosinophilia and rearrangements of PDGFRA, PDGFRB, and FGFR1 were recognized as a standalone category in the 2008 WHO classification. Subsequently, PCM1-JAK2 was added to this family as a new provisional entity in the 2016 WHO classification (1). Besides the rare cases of SM associated with a myeloid/lymphoid neoplasm with eosinophilia, peripheral eosinophilia may affect up to $28 \%$ of all SM patients (2). A paper from 2007 compared D816Vpositive SM and FIP1L1/PDGFRA-positive chronic eosinophilic leukemia (13). The distinguishing features for chronic eosinophilic leukemia included the degree of eosinophilia in relation to the tryptase level, the absence of dense MC aggregates, and pulmonary and cardiac symptoms. The authors concluded that the FIP1L1/ PDGFRA gene fusion and D816V-KIT mutation cause different clinical syndromes and a distinction is essential for therapeutic decisions (13).

Herein, we reported a case of indolent KIT D816V-positive SM with eosinophilia at diagnosis, which after two years showed an evolution to myeloid neoplasm with PDGFRA rearrangement.
At disease onset, the clinical scenario was characterized by a moderate increase of eosinophils and serum tryptase. Genetic data revealed KIT D816V mutation, while rearrangements for eosinophilia were not identified. SM and myeloid neoplasms with eosinophilia were considered in the differential diagnosis.

Similar to SM, myeloid neoplasms with eosinophilia and specific rearrangements can show dysplastic eosinophils and spindleshaped MCs. The MCs can also be CD25 positive, but do not form compact aggregates, express CD2, or carry the KIT D816V mutation (14). Our patient displayed all these characteristics; therefore a diagnosis of SM seemed to be the most likely despite a low KIT VAF.

During the follow-up, the patient complained of a worsening of flushing, headache, and eosinophilia. Detection of the FIP1L1PDGFRA mutation and absence of diagnostic criteria for SM led us to a diagnosis of myeloid neoplasm with PDGFRA rearrangement.

When the PDGFRA rearrangement was identified the eosinophilic component became predominant either in the peripheral blood and bone marrow; Pardanani et al. described 12 cases of SM and chronic eosinophilic leukemia with FIP1L1PDGFRA. These patients with eosinophilia were more likely to be males and exhibit a "loose" pattern of MC infiltration in BM trephines; 8 of them were also screened for KIT D816V and all tested negative (15).

A clonal relationship between the $\mathrm{MC}$ and the associated hematologic non-MC component has been sought using KIT and other mutations as markers of clonality $(13,14)$. Various studies conducted in patients with concomitant diagnosis of acute myeloid leukemia and SM demonstrated evidence that neoplastic MC and myeloid leukemic blasts are likely to develop from common hematopoietic progenitors $(16,17)$. Subsequent studies supported the concept of advanced SM pathogenesis as a multimutated neoplasm, in which KIT D816V mutation represents a "phenotype modifier" of clonal hematopoietic stem cell disorders toward SM (18). These findings challenged the concept that the SM and the associated non-MC-hematological disease arise uniformly from a pre- 


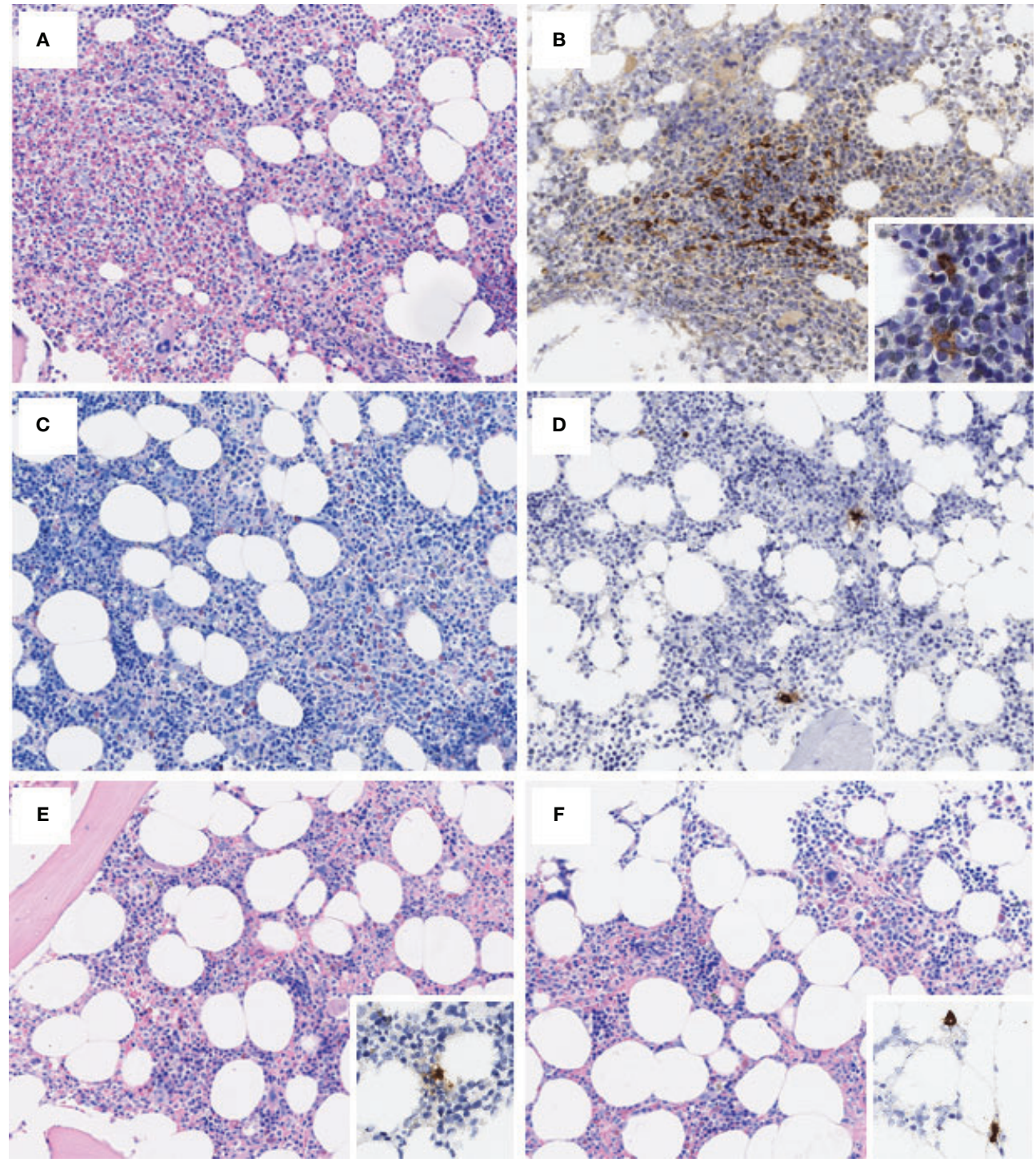

FIGURE 2 | (A) (Giemsa, 200x) depicts a hypercellular bone marrow, featuring expansion of the eosinophilic lineage, comprising maturing to fully mature eosinophils, while tryptase stain [(B); 200x] delineates the presence of scattered aggregates of epitheliod to spindled mast cells, featuring at least partial CD25-positivity (inset, 400x). Bone marrow biopsy at 3 months from Imatinib initiation [(C); Giemsa, 200x] shows a reduction of cellularity, eosinophilic compartment, and mast cells [(D); tryptase, 200x], which appear scattered. Restitutio ad integrum of the hematopoiesis is steadily apparent in subsequent biopsies [(E, F); Giemsa, 200x], with only scattered tryptase+ cells [(E, F) insets].

committed neoplastic progenitor cell harboring a KIT mutation and suggest that this category is highly heterogeneous (18).

Our case corroborates data by Maric and Pardanani $(13,15)$ who considered eosinophilic disorders and systemic mastocytosis as clinically distinct entities with different therapeutic needs. On a molecular level, further studies of next-generation and single-cell sequencing may be of benefit to clarify whether or not KIT mutation and PDGFRA rearrangement should be considered as different clones.

At the time of identification of the FIPL1-PDGFRA transcript, our patient did not meet the diagnostic criteria for SM: on bone marrow, MCs formed rare aggregates, no cytofluorimetric abnormal markers or KIT D816V mutation were identified, and serum tryptase was $20 \mathrm{ng} / \mathrm{ml}$.

According to the literature, only skin diseases in adult patients with SM could regress, in approximately $10 \%$ of the cases, while there is no evidence of spontaneous disappearance of bone marrow findings (19). In our case, the expansion of the eosinophilic component was associated with the regression of the MC counterpart, which could be masked or really disappeared during the two years after the SM diagnosis.

ISM has a nearly normal life expectancy; symptom-directed treatment should be considered in all symptomatic patients. These therapies are directed at MC degranulation symptoms, 
symptomatic skin disease, and osteopenia/osteoporosis. Cytoreductive therapy could be required for advanced SM; in this patient setting, novel agents with potent inhibitory activity against KIT demonstrated significant clinical benefit and reduction of bone marrow MC burden (2).

Initially, our patient received only symptomatic therapy directed towards osteoporosis treatment. When the myeloid neoplasm with PDGFRA rearrangement was diagnosed, low-dose imatinib was started to avoid organ damage related to eosinophilia.

The durable hematologic and molecular remissions induced by imatinib in FIP1L1-PDGFRA-positive myeloid neoplasms have been corroborated by many studies; generally, $100 \mathrm{mg}$ daily may be sufficient to achieve and maintain a long-term molecular response (7-9).

In our case report, after 3 months from the start of imatinib treatment bone marrow evaluation and peripheral blood tests showed normal findings with complete molecular remission of the FIP1L1-PDGFRA-associated neoplasm. Therapy was welltolerated, and the patient is still in complete molecular response after 18 months of therapy.

In conclusion, $\mathrm{SM}$ is a rare disease with an unpredictable clinical course, especially when another associated hematological disease is diagnosed. Little is known about the underlying molecular and immunological mechanisms that can promote one entity prevailing over the other one. Extensive clinical monitoring associated with molecular testing is essential to better define the disease and consequently its prognosis and treatment.

\section{PATIENT PERSPECTIVE}

With the diagnosis of SM, the patient seemed very concerned. He struggled to accept that only symptomatic therapy was necessary for his rare medical condition; his concern increased even more once flushing worsened, headache became recurrent, and

\section{REFERENCES}

1. Arber DA, Orazi A, Hasserjian R, Thiele J, Borowitz MJ, Le Beau MM, et al. The 2016 Revision to the World Health Organization Classification of Myeloid Neoplasms and Acute Leukemia. Blood (2016) 127(20):2391-405. doi: 10.1182/blood-2016-03-643544

2. Pardanani A. Systemic Mastocytosis in Adults: 2021 Update on Diagnosis, Risk Stratification and Management. Am J Hematol (2021) 96(4):508-25. doi: 10.1002/ajh.26118

3. Lim KH, Pardanani A, Butterfielf JH, Li CY, Tefferi A. Cytoreductive Therapy in 108 Adults With Systemic Mastocytosis: Outcome Analysis and Response Prediction During Treatment With Interferon-Alpha, Hydroxyurea, Imatinib Mesylate or 2-Chlorodeoxyadenosine. Am J Hematol (2009) 84:790-4. doi: 10.1002/ajh.21561

4. Brown LE, Zhang D, Persons DL, Yacoub A, Ponnala S, Cui W. A 26-YearOld Female With Systemic Mastocytosis With Associated Myeloid Neoplasm With Eosinophilia and Abnormalities of PDGFRB, T (4,5)(Q21;Q33). Case Rep Hematol (2016) 2016:4158567. doi: 10.1155/2016/4158567

5. Shomali W, Gotlib J. World Health Organization-Defined Eosinophilic Disorders: 2019 Update on Diagnosis, Risk Stratification, and Management. Am J Hematol (2019) 94(10):1149-67. doi: 10.1002/ajh.25617

6. Gotlib J, Cools J. Five Years Since the Discovery of FIP1L1-PDGFRA: What We Have Learned About the Fusion and Other Molecularly eosinophilia led to a diagnosis of myeloid neoplasm with PDGFRA rearrangement. The patient's perspective completely changed when the low dose imatinib was started; the treatment was well-tolerated and a complete molecular response with symptom remission was obtained.

\section{DATA AVAILABILITY STATEMENT}

The original contributions presented in the study are included in the article/supplementary material. Further inquiries can be directed to the corresponding author.

\section{ETHICS STATEMENT}

The studies involving human participants were reviewed and approved by the responsible committee on human experimentation (institutional and national). The patients/ participants provided their written informed consent to participate in this study. Written informed consent was obtained from the individual(s) for the publication of any potentially identifiable images or data included in this article.

\section{AUTHOR CONTRIBUTIONS}

MS, GC, LB, and FIG provided the patient data regarding the hematological disease. MS, GC, and FIG wrote the manuscript. $\mathrm{CE}-\mathrm{V}$ provided patient data for what concerns endocrinological aspects. GAC performed the histological examinations of bone marrow, provided iconographic materials, interpreted these data, and integrated them in the manuscript. SF and SL provided patient data regarding molecular aspects. All authors critically read and approved the final manuscript.
Defined Eosinophilias. Leukemia (2008) 22:1999-2010. doi: 10.1038/ leu.2008.287

7. Jovanovic JV, Score J, Waghorn K, Cilloni D, Gottardi E, Metzgeroth G, et al. Low-Dose Imatinib Mesylate Leads to Rapid Induction of Major Molecular Responses and Achievement of Complete Molecular Remission in FIP1L1PDGFRA-Positive Chronic Eosinophilic Leukemia. Blood (2007) 109:463540. doi: 10.1182/blood-2006-10-050054

8. Baccarani M, Cilloni D, Rondoni M, Ottaviani E, Messa F, Merante S, et al. The Efficacy of Imatinib Mesylate in Patients With FIP1L1-PDGFRalphaPositive Hypereosinophilic Syndrome. Results of a Multicenter Prospective Study. Haematologica (2007) 92:1173-9. doi: 10.3324/haematol.11420

9. Metzgeroth G, Schwaab J, Naumann N, Jawhar M, Haferlach T, Fabarius A, et al. Treatment-Free Remission in FIP1L1-PDGFRA-positive Myeloid/ Lymphoid Neoplasms With Eosinophilia After Imatinib Discontinuation. Blood Adv (2020) 4(3):440-3. doi: 10.1182/bloodadvances.2019001111

10. Da Silva EZ, Jamur MC, Oliver C. Mast Cell Function: A New Vision of an Old Cell. J Histochem Cytochem (2014) 62(10):698-738. doi: 10.1369/ 0022155414545334

11. Laine E, Chauvot de Beauchêne I, Perahia D, Auclair C, Tchertanov L Mutation D816V Alters the Internal Structure and Dynamics of C-KIT Receptor Cytoplasmic Region: Implications for Dimerization and Activation Mechanisms. PloS Comput Biol (2011) 7(6):e1002068. doi: 10.1371/ journal.pcbi. 1002068 
12. Erben P, Schwaab J, Metzgeroth G, Horny HP, Jawhar M, Sotlar K, et al. The KIT D816V Expressed Allele Burden for Diagnosis and Disease Monitoring of Systemic Mastocytosis. Ann Hematol (2014) 93(1):81-8. doi: 10.1007/s00277013-1964-1

13. Maric I, Robyn J, Metcalfe DD, Fay MP, Carter M, Wilson T, et al. KIT D816V-Associated Systemic Mastocytosis With Eosinophilia and FIP1L1/ PDGFRA-Associated Chronic Eosinophilic Leukemia Are Distinct Entities. J Allergy Clin Immunol (2007) 120(3):680-7. doi: 10.1016/ j.jaci.2007.05.024

14. Klion AD, Noel P, Akin C, Law MA, Gilliland DG, Cools J, et al. Elevated Serum Tryptase Levels Identify a Subset of Patients With a Myeloproliferative Variant of Idiopathic Hypereosinophilic Syndrome Associated With Tissue Fibrosis, Poor Prognosis, and Imatinib Responsiveness. Blood (2003) 101 (12):4660-6. doi: 10.1182/blood-2003-01-0006

15. Pardanani A, Lim KH, Lasho TL, Finke C, McClure RF, Li C, et al. Prognostically Relevant Breakdown of 123 Patients With Systemic Mastocytosis Associated With Other Myeloid Malignancies. Blood (2009) 114(18):3769-72. doi: 10.1182/blood-2009-05-220145

16. Fritsche-Polanz R, Fritz M, Huber A, Sotlar K, Sperr WR, Mannhalter C, et al. High Frequency of Concomitant Mastocytosis in Patients With Acute Myeloid Leukemia Exhibiting the Transforming KIT Mutation D816V. Mol Oncol (2010) 4(4):335-46. doi: 10.1016/j.molonc.2010.04.008

17. Pullarkat V, Bedell V, Kim Y, Bhatia R, Nakamura R, Forman S, et al. Neoplastic Mast Cells in Systemic Mastocytosis Associated With T(8;21) Acute Myeloid Leukemia Are Derived From the Leukemic Clone. Leuk Res (2007) 31(2):261-5. doi: 10.1016/j.leukres.2006.03.006
18. Jawhar M, Schwaab J, Schnittger S, Sotlar K, Horny HP, Metzgeroth G, et al. Molecular Profiling of Myeloid Progenitor Cells in Multimutated Advanced Systemic Mastocytosis Identifies KIT D816V as a Distinct and Late Event. Leukemia (2015) 29(5):1115-22. doi: 10.1038/leu.2015.4

19. Brockow K, Scott LM, Worobec AS, Kirshenbaum A, Akin C, Huber MM, et al. Regression of Urticaria Pigmentosa in Adult Patients With Systemic Mastocytosis: Correlation With Clinical Patterns of Disease. Arch Dermatol (2002) 138(6):785-90. doi: 10.1001/archderm.138.6.785

Conflict of Interest: The authors declare that the research was conducted in the absence of any commercial or financial relationships that could be construed as a potential conflict of interest.

Publisher's Note: All claims expressed in this article are solely those of the authors and do not necessarily represent those of their affiliated organizations, or those of the publisher, the editors and the reviewers. Any product that may be evaluated in this article, or claim that may be made by its manufacturer, is not guaranteed or endorsed by the publisher.

Copyright (c) 2021 Sciumè, Ceparano, Eller-Vainicher, Fabris, Lonati, Croci, Baldini and Grifoni. This is an open-access article distributed under the terms of the Creative Commons Attribution License (CC BY). The use, distribution or reproduction in other forums is permitted, provided the original author(s) and the copyright owner(s) are credited and that the original publication in this journal is cited, in accordance with accepted academic practice. No use, distribution or reproduction is permitted which does not comply with these terms. 